\title{
A Case of Sigmoid Colon Tuberculosis Mimicking Colon Cancer
}

\author{
Seong-Min Yu, Jong-Hwan Park, Min-Dae Kim, Hee-Ryong Lee, Peel Jung, Tae-Hyun Ryu, \\ Seung-Ho Choi, Il-Seon Lee ${ }^{1}$ \\ Departments of Internal Medicine and ${ }^{1}$ Pathology, Bongseng Memorial Hospital, Busan, Korea
}

Tuberculosis of the sigmoid colon is a rare disorder. An 80-year-old man visited Bongseng Memorial Hospital for medical examination. A colonoscopy was performed, and a lesion in the sigmoid colon that was suspected to be colon cancer was found. A biopsy was performed, and tuberculous enteritis with chronic granulomatous inflammation was diagnosed. Intestinal tuberculosis is most frequent in the ileocecal area, followed by the ascending colon, transverse colon, duodenum, stomach, and sigmoid colon, in descending order. Hence, we report a case of intestinal tuberculosis in the sigmoid colon, which is rare and almost indistinguishable from colon cancer.

Keywords: Tuberculosis, Gastrointestinal; Sigmoid colon; Colonic neoplasms

\section{INTRODUCTION}

Intestinal tuberculosis accounts for the majority of extrapulmonary tuberculosis. Due to its nonspecific symptoms, it is, however, difficult to discriminate from other intestinal diseases. Intestinal tuberculosis should be diagnosed based on overall considerations, including clinical manifestations, colonoscopy findings, and biopsy. Intestinal tuberculosis mainly invades the ileocecal area and is presented as a transverse ulcer. Thus, it must be discriminated from Crohn's disease, ulcerative colitis, colon cancer, ischemic colitis, amoeba, periappendiceal tumors, and lymphomas. The recent developments in colonoscopy, biopsies and acid-fast bacilli (AFB) smears can enable an early diagnosis [1]. In this case study, the authors report a case of intestinal tuberculosis which was presented as a hypertrophic ulcer, mimicking colon cancer in the sigmoid colon (SC).

Received: July 13, 2012 - Accepted: September 8, 2012

Correspondence to: Jong-Hwan Park, M.D.

Department of Internal Medicine, Bongseng Memorial Hospital,

401 Jungang-daero, Dong-gu, Busan 601-723, Korea

Tel: +82-51-664-4210, Fax: +82-51-664-4209

E-mail: drjohn21@daum.net

(C) 2012 The Korean Society of Coloproctology

This is an open-access article distributed under the terms of the Creative Commons Attribution NonCommercial License (http://creativecommons.org/licenses/by-nc/3.0) which permits unrestricted noncommercial use, distribution, and reproduction in any medium, provided the original work is properly cited.

\section{CASE REPORT}

An 80-year-old male patient visited Bongseng Memorial Hospital for a regular medical examination. The patient had a blood pressure of 110/70 $\mathrm{mmHg}$, a heart rate of 80 beats/min, a respiratory rate of 24 times $/ \mathrm{min}$, and a body temperature of $36.8^{\circ} \mathrm{C}$. No particular symptoms were identified, and he had normal consciousness. Auscultation revealed that the heart sound was normal, but a crackle was observed in both lower lungs. Peripheral blood tests showed white blood cell, 5,700 $\mathrm{mm}^{2}$; neutrophils, 58.4\%; hemoglobin, $13.4 \mathrm{~g} / \mathrm{dL}$; mean corpuscular volume, $38.8 \%$; and platelets, $218,000 \mathrm{~mm}^{2}$. Blood biochemistry showed total protein, $7.3 \mathrm{~g} / \mathrm{dL}$; globulin, $3.2 \mathrm{~g} / \mathrm{dL}$; total billirubin, $0.9 \mathrm{mg} / \mathrm{dL}$; serum glutamic oxalacetic transaminase, $20 \mathrm{IU} / \mathrm{L}$; serum glutamic pyruvic transaminase, $9 \mathrm{IU} / \mathrm{L}$; alkaline phosphatase, $288 \mathrm{IU} / \mathrm{L}$; and lactic dehydrogenase, 293 IU/L.

Colonoscopy showed an irregular inflammatory lesion in the ascending colon, and an ulcerative mass that covered more than $1 / 3$ of the intestinal tract was observed in the SC $15 \mathrm{~cm}$ superiorly to the anal verge. The ulcerative mass in the $\mathrm{SC}$ was suspected as a malignant lesion in the initial tests, so a biopsy was performed (Fig. 1A). An abdominal computed tomography (CT) showed that no particular clinical finding, except for a slight enlargement of the intestinal wall of the ileocecal area, lymphadenopathy, multiple cysts in the liver, 1-cm gallstones, and gallbladder wall distension.

A chest X-ray (Fig. 2A) showed an increase in opacity in the bi- 
Journal of The Korean Society of A Case of Sigmoid Colon Tuberculosis Mimicking Colon Cancer

Coloproctology Seong-Min Yu, et al.

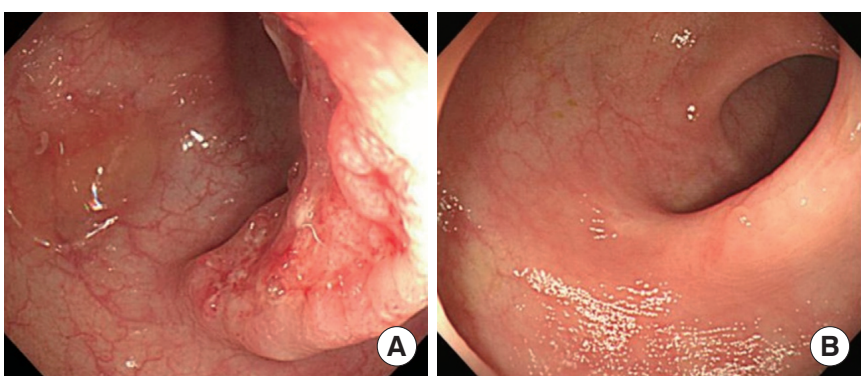

Fig. 1. Colonoscopic findings. (A) Sigmoid colon shows a circular ulcerative mass-like lesion. An endoscopic biopsy was performed. (B) An improved ulcerative lesion and small polyp (9-month follow-up).
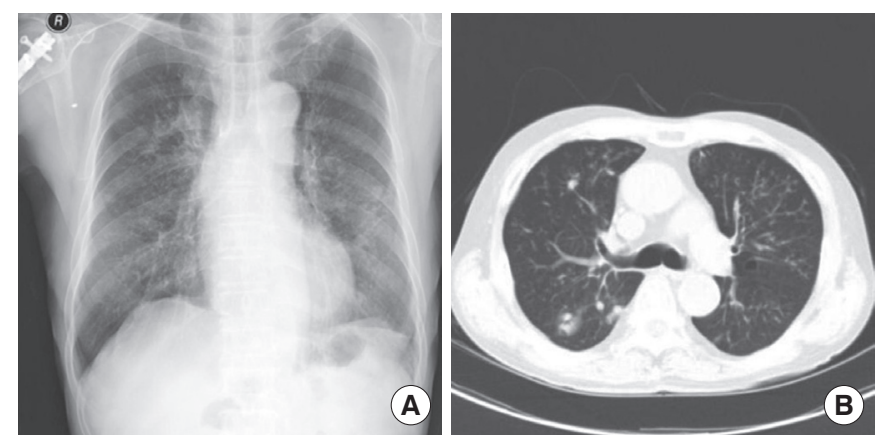

Fig. 2. (A) Chest X-ray shows increased opacity of both lower lungs. (B) Chest computed tomography findings show nodules and subtle centrilobular nodules in both lungs.

lateral lower lung, and a chest CT (Fig. 2B) showed bilateral pulmonary emphysema, an endobrachial nodule, and a pulmonary nodule. Thus, radiological findings were reasonable for the assumption of active tuberculosis. In addition, an AFB trace was found in a sputum smear. The result of the biopsy showed that the inflammatory lesion in the ascending colon was chronic colitis, and that the lesion in the SC was chronic granulomatous inflammation (Fig. 3).

As colon cancer was endoscopically suspected, a follow-up colonoscopy was conducted one month after anti-tuberculous medication. As a result, the ulcerative mass observed in the SC was improved, and inflammatory polyps were observed in the uncreative area (Fig. 1B). A biopsy was additionally conducted, and chronic inflammation was the only finding. A chest X-ray showed that the opacity of both lower lungs had decreased. The patient's respiratory symptom was also improved. An improvement was confirmed in a follow-up colonoscopy conducted 6 months later. At present, the patient has completely recovered via anti-tuberculous medication for 9 months and has been following an outpatient follow-up schedule.

\section{DISCUSSION}

According to a World Health Organization report (1990), 8 mil-

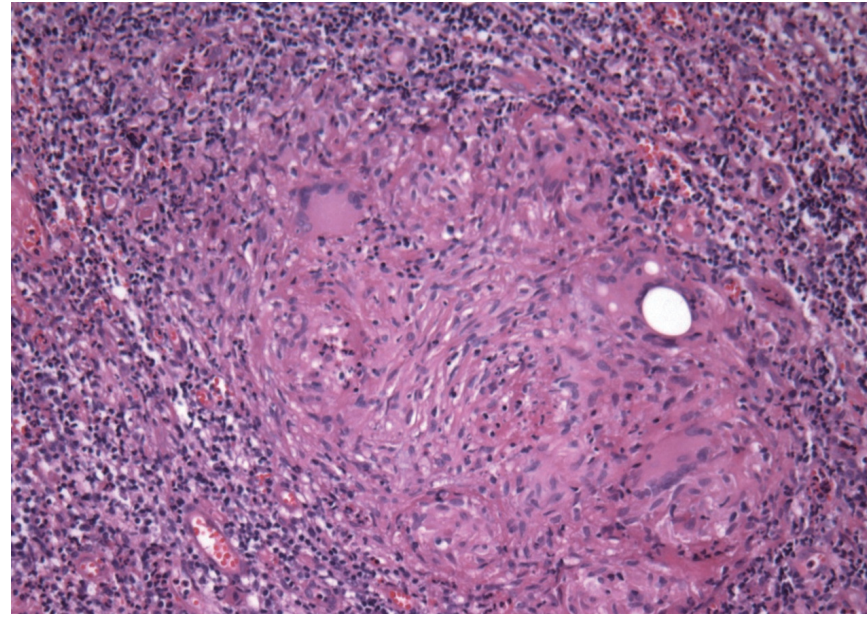

Fig. 3. Histological findings of the colon. Colonoscopic biopsy shows granulomatous inflammation and giant cells $(\mathrm{H} \& \mathrm{E}, \times 200)$.

lion patients are estimated to be diagnosed with tuberculosis annually, among whom approximately 3 million die of tuberculosis [1]. The annual report on the cause of death statistics (2005) reported that the Korean death rate due to tuberculosis was 5.9 per 100,000 people, which is higher than that of Europe (0.3), the United States $(0.8)$ or Japan (1.7). The incidence of tuberculosis patients in their 20s was 88.7 per 100,000 people in Korea, which showed a high morbidity rate.

In Korea, extrapulmonary tuberculosis has incidence rates of $26.5 \%, 17.8 \%$, and $9.7 \%$ for the pleura, lymph nodes, the intestine/ peritoneum in that order [2]. Considering a foreign study reporting that $46 \%$ of pulmonary tuberculosis patients also had intestinal tuberculosis or a domestic study reporting that $54 \%$ of active tuberculosis patients had intestinal tuberculosis on colonoscopy, the actual rate of intestinal tuberculosis could be even higher. In particular, as immune-compromised patients, acquired immune deficiency syndrome patients, and patients with multi-drug-resistant tuberculosis have been increasing, the incidence rate of intestinal tuberculosis has also been increasing in western countries [3]. Intestinal tuberculosis frequently occurs in adults, and its maleto-female ratio is 1:2. It has generally nonspecific symptoms, which include chronic abdominal pain, weight loss, diarrhea, nausea, vomiting, and fever. It has physical findings such as chronic signs of illness, malnutrition, palpable mass, tenderness, and distended abdomen [4]. It is thought to be mainly caused by the swallowing of infected sputum, hematogenous transmission of active tuberculosis, direct invasion of mycobacterium tuberculosis contained in milk or other foods entering the intestine, or as secondary tuberculosis by mycobacterium tuberculosis from other organs [5].

Intestinal tuberculosis should be diagnosed via an overall consideration, including clinical manifestations, colonoscopic findings, and biopsy. In general, intestinal tuberculosis has been known to most frequently invade the ileocecal area. In addition, it invades the ascending colon, colon, appendix, duodenum, stomach, and 
$\mathrm{SC}$ in that order [6]. The gross endoscopic findings of intestinal tuberculosis are mainly divided into three groups: multiple superficial ulcerative lesions (60\%), hypertrophic lesions characterized as scars, fibrosis, and pseudotumors (10\%), and mixed hypertrophic lesions accompanied by ileocecal hypertrophy and ulceration (30\%) [4]. In addition, endoscopic findings showed that the involvement of fewer than four segments, pseudopolyps, ulcer scars, and an open ileocecal valve were also observed in intestinal tuberculosis. Discriminative conditions include Crohn's disease, ulcerative colitis, colon cancer, ischemic colitis, amoebas, periappendiceal tumors, and lymphomas [7]. The diagnostic rate of intestinal tuberculosis may increase via biopsy identification of a caseating granuloma or $\mathrm{AFB}$, or through the detection of additional mycobacterium tuberculosis cultures [8].

Biopsies of patients with intestinal tuberculosis show various positive rates, such as the positive rate of a granuloma (41 to 48\%) [8], the positive rate of a caseating granuloma (8 to $18 \%$ ) [9], the positive rate of AFB staining (0 to 100\%) [9], and the positive rate of mycobacterium tuberculosis cultures (0 to 69\%) [9]. The diagnostic rate of intestinal tuberculosis increases if an active tuberculous lesion is observed in other regions when intestinal tuberculosis is suspected. A domestic study reported that active tuberculosis (29.8\%), tuberculous peritonitis (9.3\%), lymphadenitis (4.0\%), and tuberculous pleurisy (2.6\%) were accompanied by other forms of tuberculosis [10].

In this study, a nonspecific ulcerative lesion in the ascending colon and a hypertrophic ulcer lesion that required discrimination from colon cancer in the SC were shown, and chronic granulomatous lesions were observed in a biopsy conducted on the SC. In addition, active tuberculosis was observed to have invaded the lung through chest X-ray and CT.

For the treatment of intestinal tuberculosis, a 6- to 9-month shortterm therapy is effective, and most cases of intestinal tuberculosis are effectively treated with standard antituberculous medication. When intestinal tuberculosis is suspected, a response to the treatment with antituberculous medication is also helpful for diagnosis. A colonoscopic follow-up should be conducted 2 to 3 months after anti-tuberculous medication. In this case study, as colon cancer was initially suspected based on an endoscopic examination, a colonoscopy was conducted one month after drug medication, and a good outcome was achieved.

Intestinal tuberculosis frequently occurs in the ileocecal area, but rarely occurs in the SC. The reason for its frequent occurrence in the ileocecal area is due to the physiological stasis of the ileoce$\mathrm{cal}$ area and the abundance of lymphatic tissues around it [11]. Few previous studies have reported on tuberculosis that occurs in the SC, and three cases of intestinal tuberculosis accompanied by colon cancer were previously reported. In all three cases, the intestinal stasis seemed to contribute somewhat to the occurrence of intestinal tuberculosis, as there was a luminal narrowing due to the mass lesion. In this case study, however, lymphadenopathy and intestinal stenosis were not found. Thus, the reason for the occurrence of intestinal tuberculosis is not clear. In addition, it was difficult to discriminate intestinal tuberculosis with colon cancer, as the intestinal tuberculosis of this case study was a hypertrophic ulcer rather than the generally-observed linear transverse ulcer. The authors diagnosed nontypical intestinal tuberculosis that occurred in the SC and successfully treated it with anti-tuberculous medication.

\section{CONFLICT OF INTEREST}

No potential conflict of interest relevant to this article was reported.

\section{REFERENCES}

1. Kim SK. Overview of recent tuberculosis. Med Postgrad 1993;21: 305-11.

2. World Health Organization. Global tuberculosis control: a short update to the 2009 report [Internet]. Geneva: World health Organization, c2012 [cited 2012 May 10]. Available from: http://www. who.int/tb/features_archive/globalreport09_update_8dec09/en/ index.html.

3. Mehta JB, Dutt A, Harvill L, Mathews KM. Epidemiology of extrapulmonary tuberculosis. A comparative analysis with pre-AIDS era. Chest 1991;99:1134-8.

4. Haubrich WS, Schaffner F, Berk JE. Intestinal tuberculosis. In: Bochus Gastroenterology. vol. 2. 5th ed. Phildelphia: W.B. Saunders Company; 1995. p. 3404-6.

5. Kapoor VK. Abdominal tuberculosis. Postgrad Med J 1998;74: 459-67.

6. Nugent FW, Kolack PF. Tuberculosis. In: Kirsner JB, editor. Inflammatory bowel disease. vol. 1. 3th ed. Philadelphia: Lea \& Febiger; 1988. p. 412-4.

7. Al Karawi MA, Mohamed AE, Yasawy MI, Graham DY, Shariq S, Ahmed AM, et al. Protean manifestation of gastrointestinal tuberculosis: report on 130 patients. J Clin Gastroenterol 1995;20: 225-32.

8. Bhargava DK, Kushwaha AK, Dasarathy S, Shriniwas, Chopra P. Endoscopic diagnosis of segmental colonic tuberculosis. Gastrointest Endosc 1992;38:571-4.

9. Shah S, Thomas V, Mathan M, Chacko A, Chandy G, Ramakrishna BS, et al. Colonoscopic study of 50 patients with colonic tuberculosis. Gut 1992;33:347-51.

10. Lee YJ, Yang SK, Byeon JS, Myung SJ, Chang HS, Hong SS, et al. Analysis of colonoscopic findings in the differential diagnosis between intestinal tuberculosis and Crohn's disease. Endoscopy 2006;38:592-7.

11. Sharma MP, Ahuja V. Abdominal (gastrointestinal tract) tuberculosis in adult. In: Schaaf HS, Zumla A, Grange JM, editors. Tuberculosis: a comprehensive clinical reference. Edinburgh: Saunders/ Elsevier; 2009. p. 424-31. 